\title{
Simian virus 40 enhancer does not affect the tumor specificity of human heparanase gene promoter
}

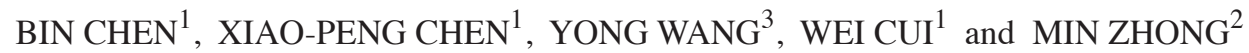 \\ ${ }^{1}$ Department of Hepatobiliary Surgery and ${ }^{2}$ Central Laboratory, Affiliated Yijishan Hospital, \\ Wannan Medical College, Wuhu, Anhui 241001; ${ }^{3}$ Department of General Surgery, \\ Pukou Traditional Chinese Medicine Hospital, Nanjin 211800, P.R. China
}

Received May 30, 2012; Accepted June 4, 2012

DOI: $10.3892 /$ br.2012.1

\begin{abstract}
The transcription activity of the tumor-specific promoter may be increased using specific DNA sequences such as simian virus 40 (SV40). Human heparanase (HPSE) gene promoter is also considered a tumor-specific promoter. However, whether or not the SV40 enhancer affects the tumor specificity of HPSE remains to be determined. The SV40 enhancer sequence, $237 \mathrm{bp}$ in length, was amplified and correctly inserted into the assigned multiple clone sites (MCS) of the eukaryotic expression vector pEGFP-Hp, which was constructed in advance. The recombinant plasmid pEGFP-HpSV40e was consistent with the anticipated Genbank data and transfected into human umbilical vein endothelial cell (ECV) and tumor cell lines, including hepatoma carcinoma (HepG2), laryngeal carcinoma (Hep2) and chronic myelogenous leukemia cell lines (K562) using lipofectamine, respectively. The expression of the reporter gene, green fluorescent protein (GFP), was detected using fluorescence microscopy and flow cytometry. The length of the amplified SV40 enchancer was $237 \mathrm{bp}$ and the sequence was in accordance with the GenBank data. The recombinant plasmid pEGFP-Hp-SV40 was consistent with the anticipated results. Fluorimetric analysis showed that the fluorescence of pEGFP-Hp-SV40e in ECV cells was as dim as pEGFP-Hp, and obviously weaker than pEGFPN1. In tumor cells including HepG2, Hep2 and K562 cells, the fluorescence of pEGFP-Hp-SV40e was similar to that of
\end{abstract}

Correspondence to: Dr Xiao-Peng Chen, Department of Hepatobiliary Surgery, Affiliated Yijishan Hospital, Wannan Medical College, Wuhu, Anhui 241001, P.R. China

E-mail:drcxp@sohu.com

Abbreviations: HPSE, heparanase; SV40, simian virus 40; GCV suicide gene, gancyclovir suicide gene; CK19, cytokerain 19; PSA, prostate specific antigen; GFP, green fluorescent protein; MCS, multiple clone site; TFBS, transcription factor binding site; TF, transcription factor

Key words: heparanase, promoter, tumor, simian virus 40 enhancer, transcription activity
pEGFP-N1, which was clearly brighter than pEGFP-Hp. The average transfecion rates in the 4 types of cells were $4.1,17.2$, 8.8 and $6.4 \%$ in the pEGFP-Hp; 18.3, 29.3, 17.0 and $13.0 \%$ in the pEGFP-Nl and 4.3, 28.8, 16.4 and $11.7 \%$ in the pEGFP-HpSV40e groups, respectively. The ratio of pEGFP-Hp-SV40e to pEGFP-Hp in all cells was 1.05, 1.67, 1.86 and 1.83, respectively. In conclusion, the inserted SV40 enhancer sequence is able to improve the transcriptional activity of the human HPSE gene promoter, but does not affect its tumor specificity.

\section{Introduction}

The tumor-specific promoter may be used to study gene targeting treatment, but the simple or natural promoter does not always meet the requirements of cancer gene therapy. One reason for this was that the activity of the partial tumorspecific promoter was weak, meaning it was difficult to induce a high expression of therapeutic genes in certain tumor cells. Modifying or transforming the structure of the promoter is crucial to accurately locate therapeutic genes and improve its expression in specific cells. Gene transcription of eukaryotic cells may be regulated by enhancers. Findings of previous studies revealed that the transcription activity of certain tumorspecific promoters was increased by enhancers. Activity of the gancyclovir (GCV) suicide gene driven by the cytokeratin 19 (CK19) promoter was markedly improved when the CK19 enhancer was added upstream of the promoter (1), and the human prostate-specific antigen (PSA) enhancer increased the expression of green fluorescent protein (GFP) regulated by the PSA promoter in prostate cancer cells (2). Therefore, the use of specific enhancers to modify the tumor-specific promoter in order to improve its transcription activity and the expression of therapeutic genes is crucial. Simian virus 40 (SV40) enhancer has been extensively applied to improve the activity of promoter, rendering it the most common molecular tool $(3,4)$.

Heparanase (HPSE) acts as an extracellular matrix catabolic enzyme that is closely correlated with tumor metastasis, angiogenesis and other diverse pathological and physiological processes (5-8). Results of previous studies have suggested that high levels of HPSE mRNA or its protein were expressed in most malignant tumors, although this expression was not found in normal somatic cells (9-12). Previously, we cloned a 561-bp-long human HPSE gene core promoter and constructed 
the eukaryotic expression plasmid pEGFP-Hp using the promoter (13). The vector was found to be expressed only in tumor cells following cell transfection. Additionally, the HPSE promoter was found to exhibit tumor specificity, and could be used for gene therapy research, although its activity was insufficient or weak (13). Therefore, modification of the HPSE promoter in order to improve its transcription activity is essential. In this study, the SV40 enhancer sequence was amplified, and inserted into the multiple clone site (MCS) downstream of the HPSE promoter in the vector pEGFP-Hp in order to construct recombinant plasmid pEGFP-Hp-SV40e. The transcription activity prior to and following modification was detected and compared, and the effect of the SV40 enhancer on the activity and tumor specificity of HPSE promoter was evaluated. The results obtained may provide an experimental basis for further research into the role played by the SV40 enhancer.

\section{Materials and methods}

Cell lines, main reagents and instruments. Normal human umbilical vein endothelial cells ECV304, human hepatocellular carcinoma cells HepG2, laryngeal epithelial cells Hep2 and positive control plasmid pEGFP-N1 were obtained from Yili Company (Hefei, China). The plasmid pEGFP-Hp was constructed and identified in our previous study (13). The SV40 enhancer-containing vector pGL3-control was purchased from Shanghai Sangon Biotech Co., Ltd. (Shanghai, China). PFX, T4 DNA ligase, restriction endonuclease EcoRI, SalI, XhoI and HindIII were all purchased from MBI Fermentas (Burlington, Ontario, Canada). The PCR purification kit, DNA gel extraction kit and a small amount of plasmid extraction kit were obtained from Daopu Company, Beijing, China. The cloning vector plasmid pMD18-T simple, DL2,000, DL5,000 and DL15,000 DNA markers, as well as dNTP and PCR buffers were purchased from Takara, Japan.

Agarose gel, tryptone and yeast extract were obtained from Oxoid, UK. Cell cultures were performed using $10 \%$ fetal bovine serum, Lipofectamine ${ }^{\mathrm{TM}}$ 2000, DMEM and RPMI-1640 media (Invitrogen, Grand Island, NY, USA). The SV40 enhancer upstream and downstream primers were synthesized by Shanghai Sangon Biotech Co., Ltd. The E. coli strain DH5 $\alpha$ was provided by Professor Xuelong Wang (Department of Parasitology, Anhui Medical University, Hefei, China). Fluorescence microscopy (Nikon, Japan) and flow cytometry (Beckman Coulter, Inc., Brea, CA, USA) were used for the subsequent analysis.

Amplification and purification of SV4O enhancer PCR. According to the GenBank DNA sequence of pGL3-control (accession no. U47296) and the principles of primer design, two primers of SV40 enhancer (forward, 5'-ATATGCgaattc CGATGGAGCGGAGAATGGG-3' and reverse, 5'-GCATGT gtcgacGCTGTGGAATGTGTGTCAGTTAGG-3') were designed using primer design software 5.0. Besides protection bases (italic letters), restriction enzyme cutting site EcoRI and SalI (lower case letters) were introduced into the 5 ' ends of primers, respectively. The sequence of the SV40 enhancer was amplified by PCR using $1 \mu 1$ pGL3enhancer DNA as a template. The reaction system was $50 \mu 1$.
The PCR conditions included initial denaturation at $94^{\circ} \mathrm{C}$ for 5 min, 35 cycles of amplification with subsequent denaturation at $94^{\circ} \mathrm{C}$ for $30 \mathrm{sec}$, annealing at $57^{\circ} \mathrm{C}$ for $50 \mathrm{sec}$, and extension for $50 \mathrm{sec}$ at $72^{\circ} \mathrm{C}$, followed by extension for $10 \mathrm{~min}$ at $68^{\circ} \mathrm{C}$. Electrophoresis was performed using $1 \%$ agarose gel. The PCR product was purified according to the manufacturer's instructions and two-way sequencing was completed by Shanghai Sangon Biotech Co., Ltd.

Identification of the SV40 enhancer. DH5a competent cells were prepared by the calcium chloride method. PCR products of SV40 enhancer and pMD18-T simple were incubated overnight for ligation, and then transformed into competent cells. Positive colonies were selected and amplified, plasmid DNA was extracted by the alkaline lysis method and identified by double digestion with EcoRI and SalI. After 1\% agarose gel electrophoresis, the positive recombinant plasmid was sent to Shanghai Sangon Biotech Co., Ltd. for sequencing.

Construction and identification of the recombinant plasmid pEGFP-Hp-SV40e. The PCR products of SV40 enhancer were digested by double digestion with EcoRI and SalI. The digested PCR product was analysed using agarose gel electrophoresis and the large fragment was retrieved. The purified product was the DNA fragment of SV40 enhancer. Plasmid pEGFP-Hp was also digested by double digestion and the large fragment was retrieved. During a ligation reaction, the two recycled products were incubated overnight at $4^{\circ} \mathrm{C}$ to insert the DNA fragment of the SV40 enhancer into MCS of the pEGFP-Hp vector. After competent cell suspension was thawed at room temperature, ligation products were added for the transformation test. The recombinant plasmid was screened, and the plasmid DNA was extracted by the alkaline lysis method, then identified by single digestion with BamHI, PCR amplification and DNA sequencing, respectively. The positive recombinant plasmid was designated as pEGFP-Hp-SV40e.

Functional analysis of the recombinant plasmid vector pEGFP-Hp-SV4Oe. ECV, HepG2, Hep2 and K562 cells were cultured in 96-well plates in RPMI-1640 cell culture medium with $10 \%$ fetal calf serum (FCS) and incubated at $37^{\circ} \mathrm{C}$ in a humidified atmosphere containing $5 \% \mathrm{CO}_{2}$. For each well, at $70-80 \%$ confluence, cells were inoculated into 3 wells at a density of $5 \times 10^{5}-1 \times 10^{6}$ cells/well and transfected with the plasmid of pEGFP-Hp, positive control plasmid pEGFP-N1 and recombinant plasmid pEGFP-Hp-SV40e, respectively. The procedure was undertaken according to the instructions for Lipofectamine $^{\mathrm{TM}}$ 2000. The cells were subsequently cultured for $48 \mathrm{~h}$, and the suspension cells were collected. The adherent cells were digested by trypsin $(0.2 \%)$. The digestion was terminated by adding blood serum culture medium. Fluorescence intensity was observed using fluorescence microscopy, and the percentage of light-emitting cell and transfection efficiency were determined by flow cytometry. Experiments were repeated at least 3 times.

\section{Results}

PCR amplification of SV40 enhancer. Agarose gel electrophoresis of PCR products of the SV40 enhancer showed a specific 


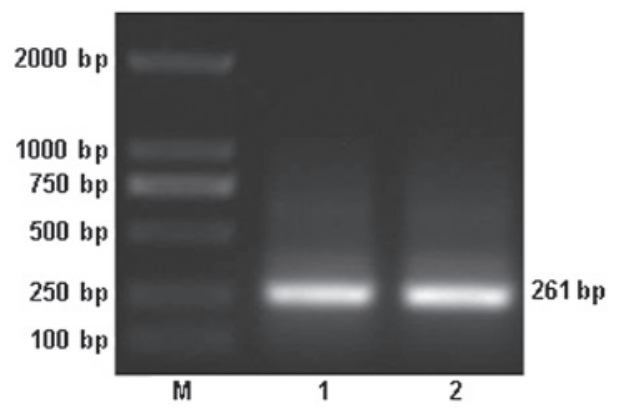

Figure 1. Electrophoretogram of PCR products where a specific band of 200500 bp was detected. M, 2,000-bp marker; lanes 1 and 2, SV40 enhancer.

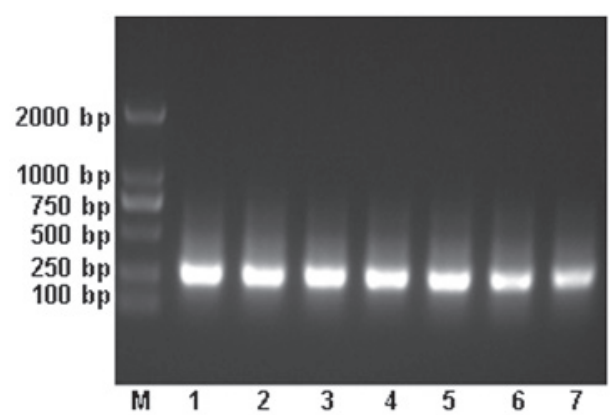

Figure 2. Electrophoretogram of PCR products of positive bacterial colony. M, 2,000-bp marker; lanes 1, 2, 3, 4, 5, 6 and 7, SV40 enhancer.

band of 250-500 bp, which was consistent with the theoretical length of $261 \mathrm{bp}$, including 24-bp enzyme cutting sites and protection bases (Fig. 1).

Identification of SV4O enhancer. After PCR amplification of positive colonies, the electrophoretogram also showed a 261-bp-long band (Fig. 2). The sequencing results of the identified positive plasmid showed that the 237 bp sequence from 46 to 282 bp was consistent with the GenBank database of pGL3-control (accession no. U47296). The sequences from

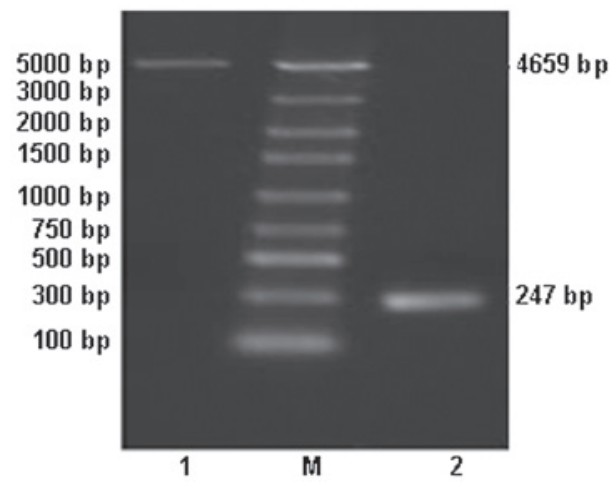

Figure 4. Electrophoretogram of plasmid pEGFP-Hp and SV40 enhancer digested with EcoRI and SalI. M, 5,000-bp marker; lane 1, pEGFP-Hp; lane 2, SV40 enhancer.

40 to 45 bp and from 283 to 288 bp were restriction enzyme cutting sites of EcoRI and SalI, respectively. No base transition, transversion, insertion and deletion were found (Fig. 3).

Construction and identification of the recombinant plasmid pEGFP-Hp-SV4Oe. The lengths of previous plasmid pEGFPHp and SV40 enhancer were 4,673 and 261 bp, respectively. Following digestion with EcoRI and SalI, two fragments were generated, however, the electrophoretogram only showed the longer fragments, of 4,659 and $247 \mathrm{bp}$, respectively. Since the cut 14-bp nucleotides moved out of the gel, it could not be found in the electrophoretogram (Fig. 4). By using the two long fragments, recombinant plasmid pEGFP-Hp-SV40e was constructed.

Recombinant plasmid pEGFP-Hp-SV40e was identified by double digestion with EcoRI and/or SalI. Two fragments were detected in the electrophoresis when the recombinant plasmid pEGFP-Hp-SV40e was digested by double digestion with EcoRI and SalI. Only a single fragment was found if the plasmid was digested with EcoRI or SalI. This finding proved that the recombinant plasmid was correctly constructed.

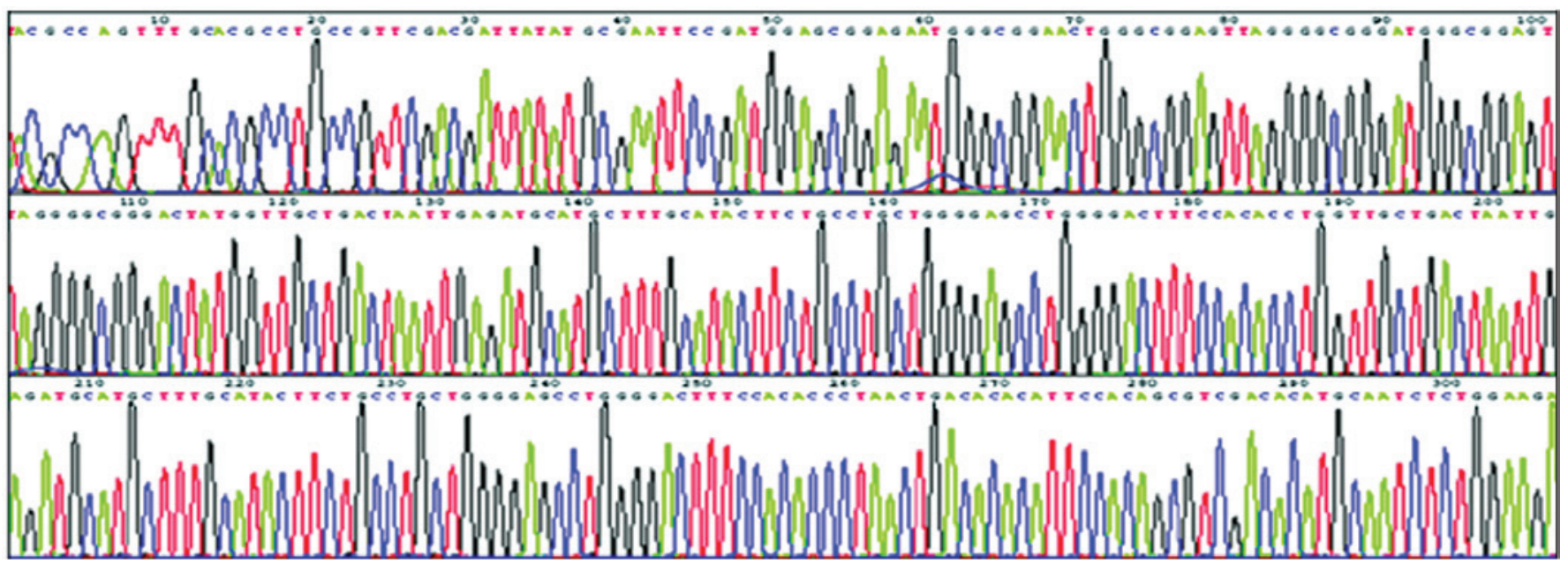

Figure 3. Partial DNA sequencing graph of SV40 enhancer shows the restriction enzyme cutting sites, but no base transition, transversion, insertion and deletion were found. 


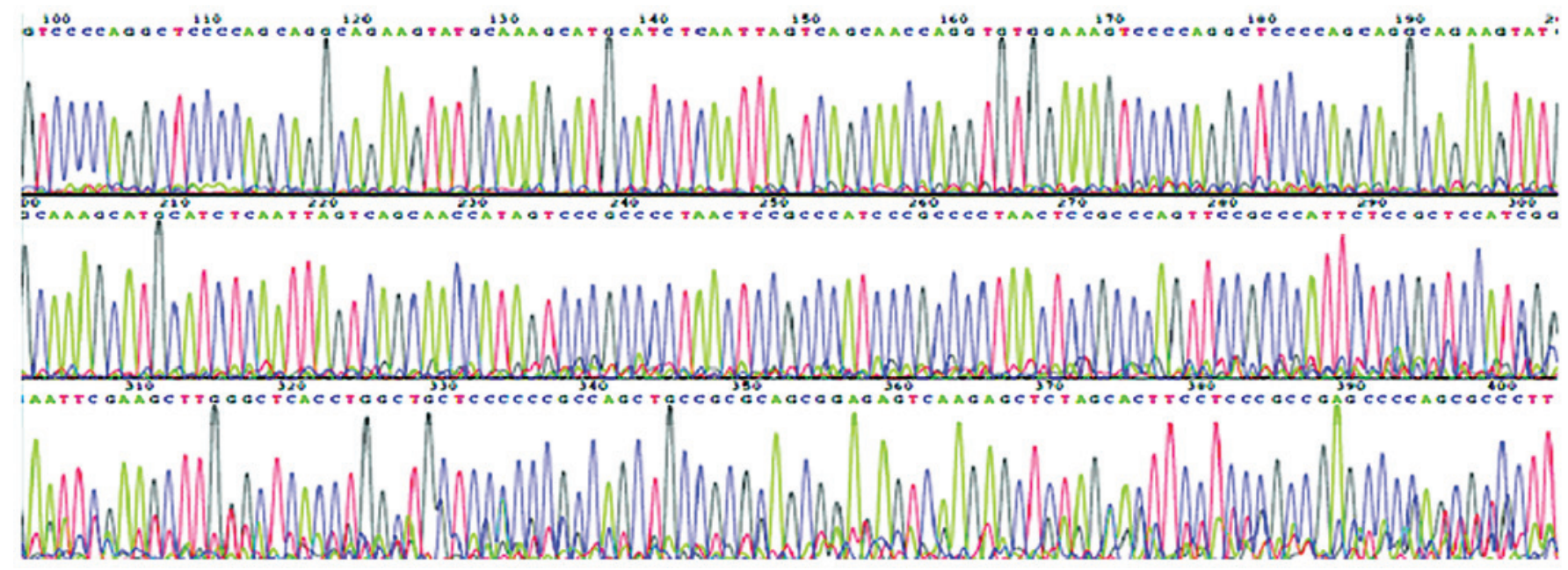

Figure 5. Partial sequencing graph of pEGFP-Hp-SV40e. The bases from 65 to $301 \mathrm{bp}$ are the SV40 enhancer sequence.
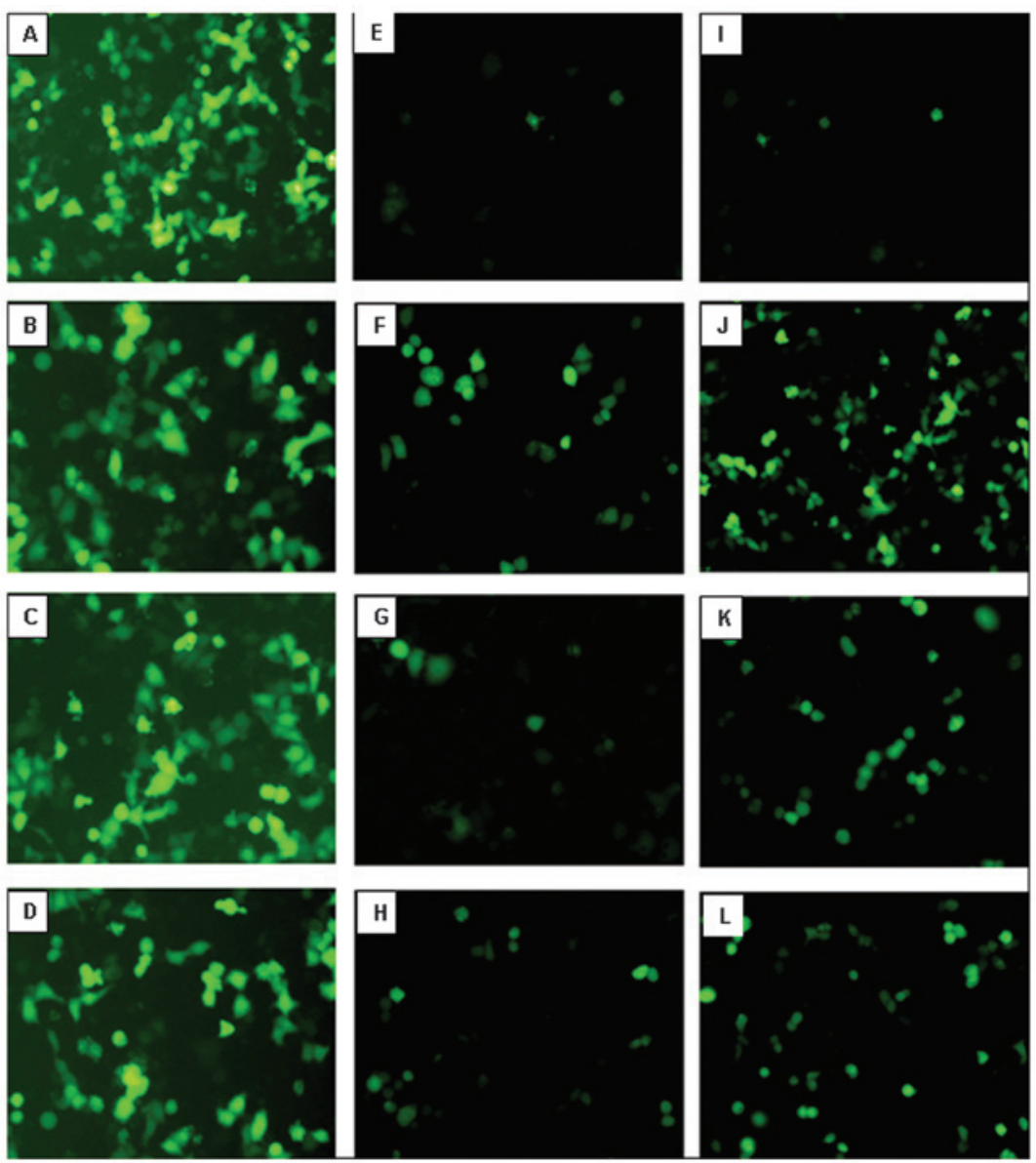

Figure 6. GFP expression of pEGFP-N1, pEGFP-Hp and pEGFP-Hp-SV40e in ECV, HepG2, Hep2 and K562 cells. The fluorescence intensity of recombinant plasmid pEGFP-Hp-SV40e in normal ECV cells was weak while the fluorescence intensity in the 3 types of tumor cells was stronger than pEGFP-Hp and similar to pEGFP-N1 (A-D) Plasmid pEGFP-N1 transfection in ECV, HepG2, Hep2 and K562 cells; (E-H) plasmid pEGFP-Hp transfection in ECV, HepG2, Hep2 and K562 cells; (I-L) plasmid pEGFP-Hp-SV40e transfection in ECV, HepG2, Hep2 and K562 cells.

When plasmid pEGFP-Hp-SV40e was used as a template, the SV40 enhancer fragment was amplified, and the sequencing results were consistent with those of the GenBank data. Following reverse sequencing, complementary sequences of the recombinant plasmid pEGFP-Hp-SV40e were obtained using Chromas Lite software. The plasmid contained a complete 237-bp SV40 enhancer sequence corresponding with the bases between 65 and $301 \mathrm{bp}$ in the sequencing graph (Fig. 5). The sequences of 59-64 and 302-307 bp were restriction enzyme cutting sites of SalI and EcoRI, respectively. The downstream sequence of the HindIII site was the DNA sequence of HPSE promoter in the vector. These findings showed that the 
SV40 enhancer sequence was properly inserted downstream of the HPSE promoter in plasmid pEGFP-Hp and that recombinant plasmid pEGFP-Hp-SV40e was successfully constructed.

Functional identification of the recombinant plasmid pEGFP-Hp-SV4Oe. Fluorescence microscopy showed that the fluorescence intensity of plasmid pEGFP-Hp was the weakest in normal ECV cells and enhanced in human tumor cell lines HepG2, Hep2 and K562. Positive control of plasmid pEGFP-N1 showed a strong fluorescence expression in all of the cells. The fluorescence intensity of recombinant plasmid pEGFP-Hp-SV40e in normal ECV cells was weak while the fluorescence intensity in the 3 types of tumor cells was stronger than that of pEGFP-Hp and similar to pEGFP-N1 (Fig. 6). Flow cytometric analysis detected the percentage of light-emitting cells of plasmid pEGFP-Hp to be only $4.1 \pm 0.3 \%$ in ECV cells; and 17.2 $\pm 1.9,8.8 \pm 1.0$ and $6.4 \pm 0.5 \%$ in HepG2, Hep 2 and K562 cells, respectively. The positive control plasmid pEGFP-Hp was found to have higher transfection rates in all cells and the percentages of light-emitting cells were 18.3 \pm 1.9 , $29.3 \pm 3.0,17.0 \pm 1.8$ and $13.0 \pm 1.2 \%$, in ECV, HepG2, Hep2 and K562 cells, respectively, suggesting that it also had a high activity in normal ECV cells. The light-emitting cell percentages of recombinant plasmid pEGFP-Hp-SV40e were 4.3 \pm 0.4 , $28.8 \pm 2.9,16.4 \pm 1.5$ and $11.7 \pm 1.2 \%$, in ECV, HepG2, Hep2 and K562 cells, respectively. The ratios of transfection rates of pEGFP-Hp-SV40e vs. pEGFP-Hp were 1.05, 1.67, 1.86 and 1.83 , in the four cell lines, respectively. These findings suggest that inserting SV40 enhancer did not affect the transfection efficiency of pEGFP-Hp in normal ECV cells, but that it is capable of increasing HPSE promoter activity in tumor cells.

\section{Discussion}

Human HPSE gene was cloned and identified almost at the same time in 1999 by several research teams (5-8). The gene, located on chromosome $4 \mathrm{q} 22$, includes 14 exons separated by 13 introns. The complete cDNA of HPSE is 1,758-bp long, and contains an open reading frame encoding a polypeptide of 543 amino acids with a calculated molecular weight of $61,192 \mathrm{Da}$. A number of studies have suggested that high levels of HPSE mRNA or protein were expressed in most malignant tumors, but no expression was detected in normal somatic cells (5-11). By degrading glycosaminoglycans, whose main component is heparan sulfate proteoglycans in extracellular matrix, HPSE plays an important role in tumor metastasis and vascularization. In recent years, HPSE has become a new therapeutic target for cancer biotherapy $(14,15)$.

The core sequence of the HPSE promoter was cloned in our previous experiment (13). Results of the sequence analysis showed that it contained the reported transcription factor binding site (TFBS) $(16,17)$ as well as the 6 E-box sites. By inserting the HPSE core promoter, we constructed a plasmid pEGFP-Hp driven by the HPSE promoter and transfected it into normal and tumor cell lines. Plasmid pEGFP-Hp was found to be expressed only in tumor cell lines and not in normal cell lines. This finding suggested that the HPSE promoter was a broad-spectrum tumor peculiar promoter and could be used as a new available tool for oncogene-targeted therapy (13). However, the detection result of microscopy and flow cytometry indicated that the GFP expression levels of pEGFP-Hp in tumor cells were lower than those of pEGFP-N1, which contains a cytomegalovirus promoter (13). The phenomenon was also observed and verified in this study. Therefore, improving the activity of HPSE promoter without changing its tumor specificity is essential.

Enhancers are usually cis-acting elements that could significantly improve the transcription efficiency of homologous or heterologous genes. Enhancer may be located upstream, downstream or within the target gene that it regulates, even at a distance from the transcription starting point. The SV40 enhancer was one of the first enhancers to exhibit strong activity. It is composed of a $72 \mathrm{bp}$ repeated sequence without obvious partiality in race and can exert a 2- to 20-fold enhancement of gene transcription in different host cells $(18,19)$. Previous results have shown that the transcription activity of human telomerase reverse transcriptase (hTERT) promoter was significantly enhanced after it was modified using SV40 enhancer (20). The mechanism of SV40 enhancer in gene regulation was associated with its Z-DNA structure. Alternating purine and pyrimidine were also detected in its repeated sequence allowing the formation of a rotating DNA structure that binds with a specific protein for transcription (21). Furthermore, SV40 enhancer promoted the ingression of plasmid vector from the cytoplasm to the nucleus to potentialize the nucleic localization of DNA that was sequence specific. For these reasons, SV40 enhancer could be applied to promote the transfection efficiency and expression of therapeutic genes.

In this study, the SV40 enhancer sequence was amplified by using the plasmid pGL3 as a template, which contained the SV40 enhancer. The sequencing result was in accordance with the GenBank data (accession no. U47296), although no insertion, deletion or substitution was evident. The result verified that the amplified SV40 enhancer sequence was correct. The plasmid pEGFP-Hp-SV40e was constructed using the amplified SV40 enhancer sequence and plasmid pEGFP-Hp, and then identified using enzyme digestion. The 237-bp SV40 enhancer was found to be successfully inserted into the appointed MCS of pEGFP-Hp by sequencing. It further proved the construction of recombinant plasmid pEGFP-Hp-SV40e was successful and consistent with the principle of molecular biology.

Following the transfection of recombinant plasmid pEGFPHp-SV40e into diverse cells, stronger fluorescence was found in 3 types of human tumor cells, using fluorescent microscopy, which was similar to the positive control plasmid pEGFP-N1. The fluorescence intensity of plasmid pEGFP-Hp was obviously weaker than the other two plasmids. Results of flow cytometry showed that the transfection efficiency of recombinant plasmid pEGFP-Hp-SV40e in the abovementioned tumor cells was similar to the control plasmid pEGFP-N1, but significantly stronger than that of pEGFP-Hp. The ratios of transfection efficiency (pEGFP-Hp-SV40e/pEGFP-Hp) in these tumor cells exceeded 1.65. These findings suggest that the inserted SV40 enhancer markedly improved the expression of HPSE promoter-driven downstream genes. The core element of the enhancer was able to combine with the endonuclear reactivator and indirectly transmit the signal from activator to transcription factor (TF), resulting in a marked increase of promoter transcription activity. Therefore, the enhancer was able to increase transcription efficiency and become an important 
component of 'gene switching' (22). We presume the inserted SV40 enhancer was capable of specifically combining with the endonuclear reactivator, thereby enhancing the transcription activity of HPSE promoter and the expression of GFP.

Findings of the present study showed that the fluorescence was extremely weak in the pEGFP-Hp-SV40e and pEGFP$\mathrm{Hp}$ groups but remained strong in the pEGFP-N1 group following transfection into the ECV cells. The transfection efficiency of pEGFP-Hp-SV40e was also low and similar to that of pEGFP-Hp. These findings suggest that the inserted SV40 enhancer does not affect the tumor specificity of HPSE promoter, although it is capable of improving HPSE promoter activity. Enhancer is a type of specific sequence in gene. Its structure is similar to that of the promoter. Following activation by the endonuclear reactivator, enhancer must specifically combine with endonuclear TF in order to improve promoter activity as well as maintain its specificity. In the plasmid pEGFP-Hp-SV40e, the function of the inserted SV40 enhancer depends on the expression of the sequence downstream of the single HPSE promoter. Therefore, the SV40 enhancer does not affect the specificity of the HPSE promoter when it enhances its activity, i.e., the recombinant plasmid pEGFP-Hp-SV40e is expressed only in tumor cells but not in normal cells. This finding is crucial to future research and application.

In conclusion, we successfully amplified a complete SV40 enhancer sequence and constructed the recombinant plasmid pEGFP-Hp-SV40e by inserting it into pEGFP-Hp. Results of the fluorescent and flow cytometric analyses found that the transfection efficiency of recombinant plasmid pEGFP-Hp-SV40e in tumor cells was significantly higher than the plasmid pEGFP-Hp, while the transfection efficiency in normal cells remained unchanged. These results demonstrate that SV40 enhancer can be used to improve HPSE promoter activity without damaging its tumor specificity. However, more studies should be carried out to assess whether or not SV40 enhancer affects the tumor specificity of the HPSE promoter when it increases transcription activity.

\section{Acknowledgements}

This study was supported by the Natural Science Foundation of Anhui, China (grant no. 11040606M181) and the Provincial Natural Science Foundation of College in Anhui, China (grant no. kj2010B239). We thank Dr Kong Lv from the Wannan Medical College (Anhui, China) for technical assistance in flow cytometry.

\section{References}

1. Ishiwata N, Inase N, Fujie T, et al: Suicide gene therapy using keratin 19 enhancer and promoter in malignant mesothelioma cells. Anticancer Res 23: 1405-1409, 2003.
2. Chapel-Fernandes S, Jordier F, Lauro F, et al: Use of the PSA enhancer core element to modulate the expression of prostateand non-prostate-specific basal promoters in a lentiviral vector context. Cancer Gene Ther 13: 919-929, 2006.

3. Li S, MacLaughlin FC, Fewell JG, et al: Muscle-specific enhancement of gene expression by incorporation of SV40 enhancer in the expression plasmid. Gene Ther 8: 494-497, 2001.

4. Li HS, Liu Y, Li DF, et al: Enhancement of DNA vaccine-induced immune responses by a 72-bp element from SV40 enhancer. Chin Med J 120: 496-502, 2007.

5. Vlodavsky I, Friedmann Y, Elkin M, et al: Mammalian heparanase: gene cloning; expression and function in tumor progression and metastasis. Nat Med 5: 793-802, 1999.

6. Hulett MD, Freeman C, Hamdorf BJ, et al: Cloning of mammalian heparanase; an important enzyme in tumor invasion and metastasis. Nat Med 5: 803-809, 1999.

7. Toyoshima M and Nakajima M: Human heparanase. Purification, characterization, cloning and expression. J Biol Chem 274: 24153-24160, 1999.

8. Kussie PH, Hulmes JD, Ludwig DL, et al: Cloning and functional expression of a human heparanase gene. Biochem Biophys Res Commun 261: 183-187, 1999.

9. El-Assal ON, Yamanoi A, Ono T, et al: The clinicopathological significance of heparanase and basic fibroblast growth factor expressions in hepatocellular carcinoma. Clin Cancer Res 7: 1299-1305, 2001.

10. Chen XP, Liu YB, Rui J, et al: Heparanase mRNA expression and point mutation in hepatocellular carcinoma. World J Gastroenterol 10: 2795-2799, 2004.

11. Komatsu N, Waki M, Sue M, et al: Heparanase expression in B16 melanoma cells and peripheral blood neutrophils before and after extravasation detected by novel anti-mouse heparanase monoclonal antibodies. J Immunol Methods 331: 82-93, 2008.

12. Ilan N, Elkin M and Vlodavsky I: Regulation, function and clinical significance of heparanase in cancer metastasis and angiogenesis. Int J Biochem Cell Biol 38: 2018-2039, 2006.

13. Chen XP, Hu LH and Cui W, et al: Activity and tumor specificity of of human heparanase gene core promoter. Mol Med Rep (In press).

14. Boyd DD and Nakajima M: Involvement of heparanase in tumor metastases: a new target in cancer therapy? J Natl Cancer Inst 96: 1194-1195, 2004

15. Yang JM, Wang HJ, Du L, et al: Screening and identification of novel B cell epitopes in human heparanase and their antiinvasion property for hepatocellular carcinoma. Cancer Immunol Immunother 58: 1387-1396, 2009.

16. Jiang P, Kumar A, Parrillo JE, et al: Cloning and characterization of the human heparanase-1 (HPR1) gene promoter. Role of GA-binding protein and Sp1 in regulating HPR1 BASAL promoter activity. J Biol Chem 277: 8989-8998, 2002.

17. de Mestre AM, Rao S, Hornby JR, et al: Early growth response gene 1 (EGR1) regulates heparanase gene transcription in tumor cells. J Biol Chem 280: 35136-35147, 2005.

18. Wasylyk B, Wasylyk C, Augereau P, et al: The SV40 72 bp repeat preferentially potentiates transcription starting from proximal natural or substitute promoter elements. Cell 32: 503-514, 1983.

19. Lee-Chen GJ and Woodworth-Gutai M: Simian virus 40 DNA replication: functional organization of regulatory elements. Mol Cell Biol 6: 3086-3093, 1986.

20. Song JS: Adenovirus-mediated suicide SCLC gene therapy using the increased activity of the hTERT promoter by the MMRE and SV40 enhancer. Biosci Biotechnol Biochem 69: 56-62, 2005.

21. Hagen FK, Zarling DA and Jovin TM: Electron microscopy of SV40 DNA cross-linked by anti-Z DNA IgG. EMBO J 4: 837-844, 1985.

22. Pennisi E: Searching for the genome's second code. Science 306: 632-635, 2004 